\title{
Characterization of PVDF/HAP Composites for Medical Applications
}

\author{
Francisco José Correa Braga, Sizue Ota Rogero ${ }^{\mathrm{a}}$, Antônio Augusto Couto ${ }^{\mathrm{a}}$, \\ Rodrigo Fernando Costa Marques ${ }^{\mathrm{b}}$, Alexandre Antunes Ribeiro ${ }^{\mathrm{c} *}$, João Sinézio de Carvalho Campos ${ }^{\mathrm{c}}$ \\ ${ }^{\mathrm{a}}$ Centro de Ciência e Tecnologia de Materiais, \\ Instituto de Pesquisas Energéticas e Nucleares - IPEN, Av. Prof. Lineu Prestes, 2242, \\ Cidade Universitária, 05508-900 São Paulo - SP, Brazil \\ ${ }^{\mathrm{b}}$ Departamento de Físico-Química, Instituto de Química, UNESP, \\ Rua Prof. Francisco Degni, s/n, CP 355, 14800-9000 Araraquara - SP, Brazil \\ 'Departamento de Tecnologia de Polímeros, Faculdade de Engenharia Química, \\ Universidade Estadual de Campinas - UNICAMP, Cidade Universitária "Zeferino Vaz", \\ CP 6066, 13081-970 Campinas - SP, Brazil
}

Received: August 14, 2006; Revised: June 22, 2007

\begin{abstract}
Biomaterials (composites and blends) play a major role in the health of modern society. This paper reports on the preparation and characterization of polyvinylidene fluoride (PVDF) and hydroxyapatite (HAP) composites, analyzing the incorporation of HAP in PVDF and investigating their mechanical properties and cytotoxicity (biocompatibility) for use in bone restoration and filling. The material was prepared in film form by the casting method. PVDF pellets were dissolved in dimethylacetamide (DMA), a HAP/DMA emulsion was prepared. The materials were mixed in proportions of 100/00, 90/10, 80/20, 70/30, 60/40, 50/50, 40/60, 30/70 in weight and left to dry in an oven, resulting in homogeneous, flexible films which were characterized by scanning electron microscopy (SEM), energy dispersive spectroscopy (EDS), X ray diffractometry (XRD), contact angle measurement, and by mechanical and cytotoxicity tests.
\end{abstract}

Keywords: polyvinylidene fluoride, polymer composites, biomaterials, hydroxyapatite

\section{Introduction}

Numerous health-related studies have revealed an ever-growing search for substances to replace bony parts lost in traumatic or nontraumatic events, and metals have been widely used in the majority of bone implants. However, the use of metallic materials in the human body has given rise to a variety of problems involving corrosion, type of wear, and/or negative tissue reaction ${ }^{1}$. In the search for bioactive protheses, improved materials containing hydroxyapatite (HAP) have aroused interest in innumerable researches ${ }^{2}$. Composite materials that favor bone formation in the injured site have been exhaustively studied. Biodegradable polymers, artificial bone cells and morphogenetic proteins with HAP loads have been investigated in the formation of natural bones ${ }^{3-7}$.

In this context, polyvinylidene fluoride (PVDF) membranes have been studied for various applications ${ }^{8-12}$, but the literature lacks reports about the use of PVDF in the regeneration of bony tissue. With regard to the anti-bacterial property of PVDF membrane ${ }^{13}$, this study investigated the maximum incorporation of HAP in this type of membrane and analyzed the results of the assays and tests considered basic for the application of PVDF/HAP composites as barriers against bone defects.

In addition to examining the physicochemical properties of these types of membranes, their biocompatibility must be ascertained since they are intended for use in bone tissue regeneration processes. The first in vitro biocompatibility test is a cytotoxicity assay. After verifying that the material is devoid of toxicity, further studies and assays must be carried out in each stage of the production process to confirm its biocompatibility up to the end product.

\section{Materials and Methods}

\subsection{Preparation of the composites}

The composites were prepared by dissolving PVDF pellets in dimethylacetamide (DMA) in a $50 \mathrm{~mL}$ glass beaker under continuous agitation and a controlled temperature of $100{ }^{\circ} \mathrm{C}$. A HAP/DMA emulsion was then prepared by agitation without heating in another $50 \mathrm{~mL}$ glass beaker. After complete dissolution of the PVDF, the HAP emulsion was added, maintaining the temperature and agitation until the mixture attained the necessary viscosity, at which point it was poured onto a $60 \mathrm{~mm}$ glass Petri dish. The mixture was then oven-dried at $110{ }^{\circ} \mathrm{C}$ for 4 hours to eliminate the DMA solvent. The resulting PVDF/HAP films, prepared in proportions of 100/00, $90 / 10,80 / 20,70 / 30,60 / 40,50 / 50,40 / 60,30 / 70$ in weight (or \%), were homogeneous and flexible.

\subsection{Characterization of the composites}

The properties of the PVDF/HAP composites were examined by scanning electron microscopy (SEM), energy dispersive spectroscopy (EDS), X ray diffractometry (XRD), contact angle measurement, and mechanical and cytotoxicity assays. The samples for mechanical and 
cytotoxicity assays were irradiated using a Co-60 gamma ray source with a dosage of $25 \mathrm{kGy}$ to ascertain the possible interference of structural modifications in their mechanical properties and to sterilize the samples for the cytotoxicity assay of biocompatibility.

\subsubsection{Scanning electron microscopy (SEM)}

Samples of PVDF/HAP composites in proportions of 100/00 and $60 / 40$ (in weight) were analyzed by SEM and EDS. The surfaces of these samples were covered with a thin carbon film to preserve the topographies of the original manufacturing process. The purpose of this analysis was to observe the heterogeneity of the surface texture by SEM (Phillips XL-30).

\subsubsection{Energy dispersive spectroscopy (EDS)}

As explained under item 2.2.1, the samples were exposed to EDS X ray of the largest possible surface area to obtain the most general representative spectrum for each sample in order to analyze its chemical elements.

\subsection{3. $\mathrm{X}$ ray diffractometry $(\mathrm{XRD})$}

Powder X ray data were collected in the $2 \theta$ interval from 23 to $100^{\circ}$, in $5 \mathrm{~s} 0.01^{\circ}$ steps, using a SIEMENS D5000 diffractometer with copper radiation monocromatized by a graphite crystal. The diffractometer was set at $40 \mathrm{kV}$ and $30 \mathrm{~mA}$.

\subsubsection{Contact angle measures}

The hanging drop method was used to measure the contact angle $(\theta)$ on the surface of PVDF/HAP samples, using a contact angle meter (TATEC Cam-micron goniometer). The contact angle measurements were taken at room temperature in $75 \%$ relative air humidity, since the $\theta$ varies only slightly with temperature, according to $\mathrm{Adam}^{14}$, who found no detectable variations in the water in several solid hydrocarbonates in a 20 to $35{ }^{\circ} \mathrm{C}$ interval. To establish the balance of the forces involved, the contact angle reading was obtained 20 seconds after deposition of the drop on the surface of the samples. Each $\theta$ value corresponds to the average of 4 measurements, with a maximum deviation of \pm 2 . The measurements were taken from samples presenting the best surface regularity, since this kind of analysis requires uniform surfaces (little roughness) to ensure the most reliable contact angle measures are obtained.

\subsubsection{Mechanical testing}

The PVDF/HAP films were tensile tested to determine their mechanical behavior. Three test specimens were tested in each of the following proportions: 100/00, 70/30 and 60/40 (in weight) and the films were tested with and without irradiation. The tensile tests were carried out in an Instron 4400 universal mechanical testing machine, with a load cell corresponding to $50 \mathrm{kgf}$, using a crosshead movement testing speed of $5 \mathrm{~mm} / \mathrm{min}$. The ultimate strength and total strain of the film were determined based on the stress-strain curves resulting from the tensile tests.

\subsubsection{Cytotoxicity assay}

The cytotoxicity assay is an in vitro method to verify the toxicity of materials. This assay was carried out according to the ISO $10993^{15}$ standard, whose methodology is described in previous papers ${ }^{16-17}$. A monolayer cell line of NCTC clone 929 obtained from the American Type Culture Collection (ATCC) was used in 96 microplate wells, to which were added serially diluted extracts of PVDF and PVDF/HAP membrane samples. The extracts were obtained by immersing the membranes in a flask containing MEM (minimum Eagle's medium) cell culture medium with $5 \%$ calf fetal serum and nonessential amino acids, which was incubated at $37^{\circ} \mathrm{C}$ for 24 hours. PVC pellets were used as negative control and the extract was obtained in the same way as the membranes. A $0.02 \%$ phenol solution was used as positive control and received the same treatment as the extract dilution. The controls served to verify the performance of the assay; a positive control must be a material that causes toxicity while a negative control must be nontoxic. The cytotoxic effect of each material was measured based on the percentage of viable cells in contact with the extract of the tested material, and quantitatively assessed by measuring the uptake of neutral red by the viable cells. The optical densities (OD) were measured in a microplate ELISA reader (Tecan Sunrise spectrophotometer) with a $540 \mathrm{~nm}$ filter, calculating the percent of cell viability in relation to the control cell. The cytotoxicity index, represented by $\mathrm{IC}_{50 \%}$ in the viability curves, was obtained by plotting the percent of viable cells against the extract concentration. The cytotoxicity index represents the concentration of extract that kills or injures $50 \%$ of the cell population in the assay.

\section{Results and Discussion}

The maximum amount of hydroxyapatite incorporated into the PVDF polymer was $40 \%$. This amount does not cause saturation of the polymeric matrix and the HAP appears on the surface of the membrane in the form of powder. In the PVDF/HAP 30/70 composition, the polymeric matrix was unable to support the high load. The film-composite was not formed and most of the HAP settled on the bottom of the glass plate.

\subsection{Scanning electron microscopy (SEM)}

Figure 1 shows SEM images magnified 500 and 5000 times. Note the PVDF polymer with a globular structure and porous regions between the globes. The PVDF/HAP composites showed the HAP phase partially filling the original porous spaces of the pure PVDF.

According to Sheldon ${ }^{18}$, the filler phase (in this case HAP) of a polymeric composite can act as a crystal-nucleating agent (or, in rare cases, as an anti-nucleant), affecting the size or the perfection of the polymer crystal. The presence of HAP to the PVDF matrix appears to modify the polymer grain formation process, altering the size and morphology of the pores.

\subsection{Energy dispersive spectroscopy (EDS)}

The EDS spectrum in Figure 2 shows the main chemical elements of the PVDF membranes of composition $100 / 00$ and 60/40. These elements were identified as carbon and fluorine originating from the PVDF structure, and as calcium $(\mathrm{Ca})$ and phosphorus $(\mathrm{P})$ originating from the incorporated HAP.

A semi-quantitative relationship of the chemical elements can be calculated based on the their peak sizes in the spectrum. The $\mathrm{Ca} / \mathrm{P}$ relationship of HAP is usually 1.65 but the one we observed was 1.15. This difference may indicate a chemical reaction between HAP and PVDF during the synthesization process. This issue can be clarified by $\mathrm{X}$ ray Diffraction and/or Fourier Transform Infrared techniques.

\section{3. $X$ ray diffractometry $(X R D)$}

The successful incorporation of HAP in the PVDF membranes was confirmed by XRD. Figure 3 shows the $X$ ray diffraction patterns of PVDF/HAP composites in the ratio of 100/00 (thick line in the graph) and 60/40 (thin line) and the HAP XRD patterns (upward pointing triangle - PDF n. 86-740). According to Lovinger ${ }^{19}$, PVDF presents Bragg peak positions at $2 \theta$ equal to $20^{\circ}, 27^{\circ}$ and $40^{\circ}$. As the diffractogram in Figure 3 demonstrates, the XRD of pure PVDF membrane presented characteristic diffraction patterns, indicating that the polymer was successfully formed. A comparison of the 

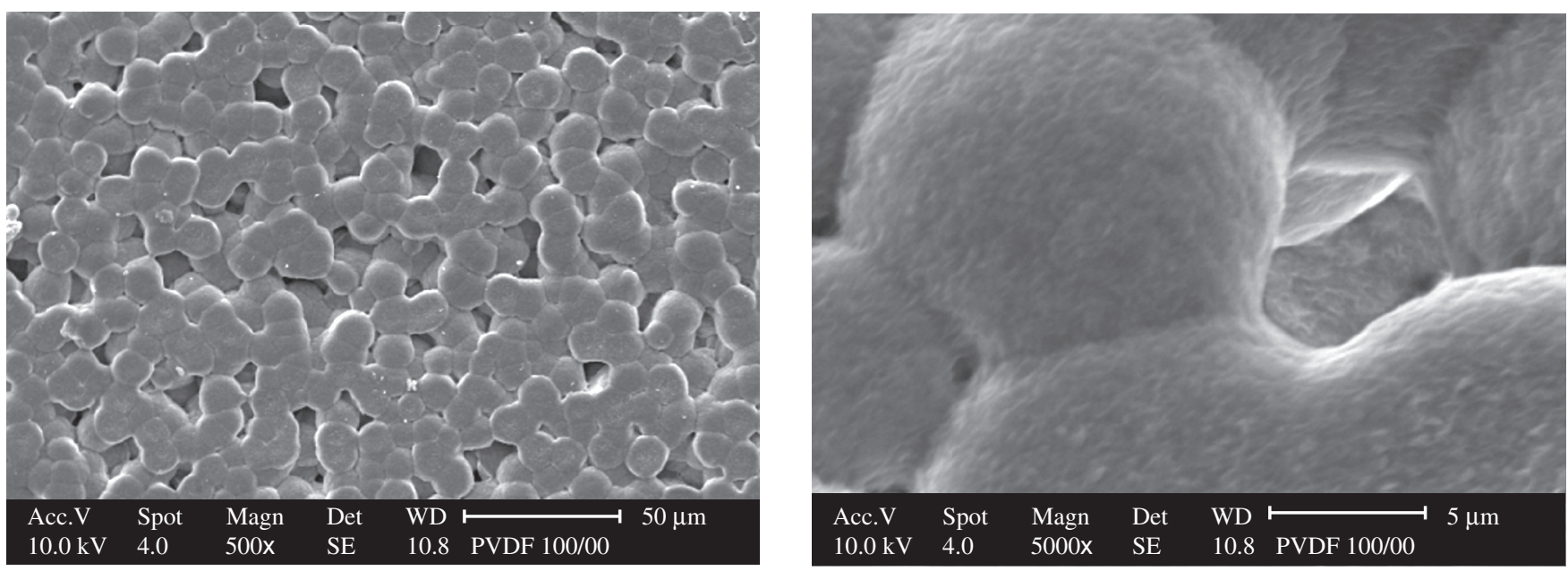

(a)
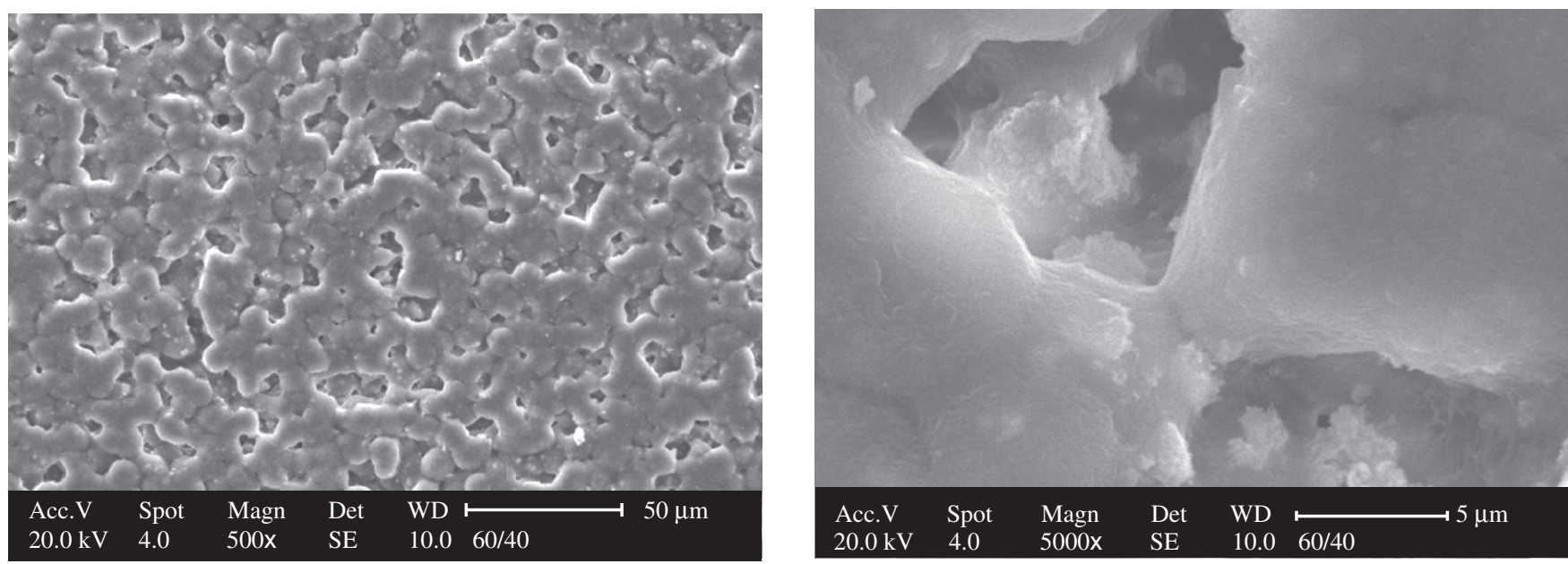

(b)

Figure 1. SEM images magnified 500 and 5000 times: a) pure PVDF membrane; and b) PVDF membrane containing $40 \%$ in weight of HAP.

Label A: 100/00

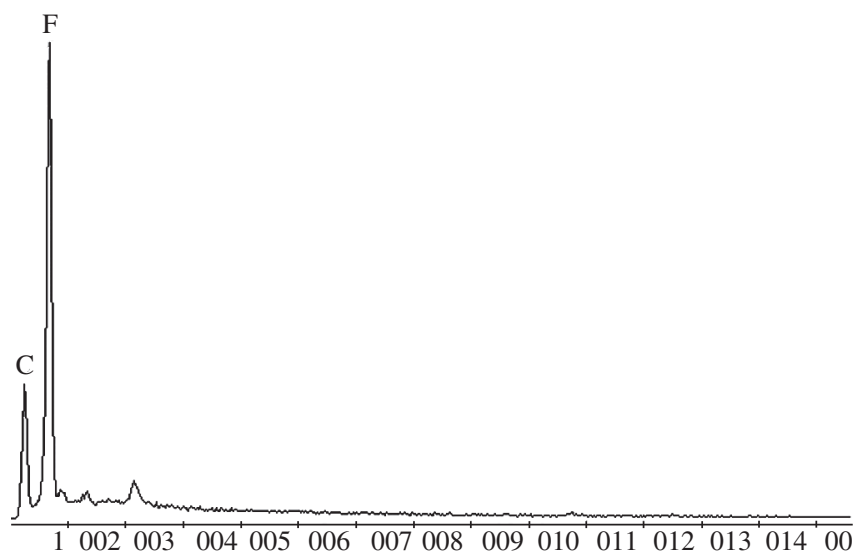

(a)
Label A: 60/40

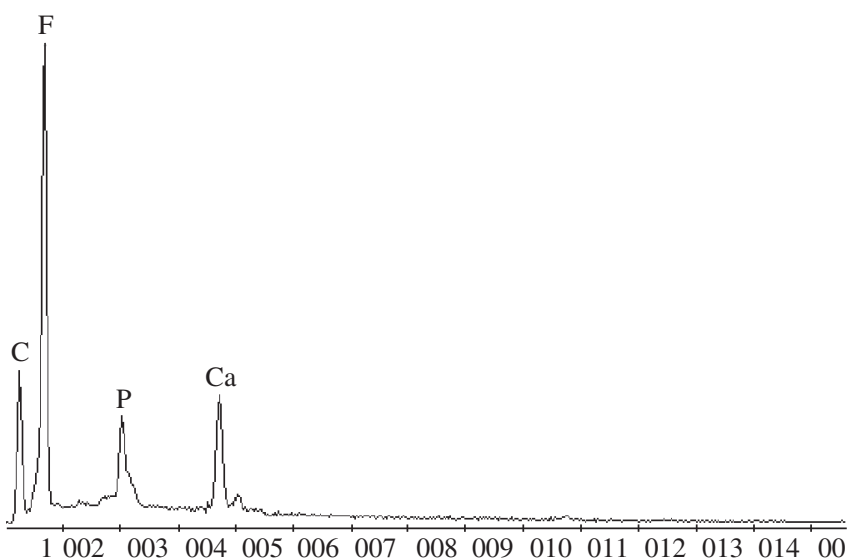

(b)

Figure 2. EDS X ray spectra: a) pure PVDF membrane; and b) PVDF membrane containing $40 \%$ in weight of HAP. 
HAP diffraction patterns (upward pointing triangle) and the XRD of PVDF/HAP 60/40 (thick line) led us to conclude that the HAP was incorporated in the PVDF membrane. The PVDF diffraction patterns remain unchanged after the membrane was impregnated with HAP, indicating that the PVDF polymeric structure was not modified during the synthesis of the composites.

\subsection{Contact angle measures}

Table 1 presents the values of the contact angles $(\theta)$ and the respective standard deviations of the PVDF/HAP composites films' surfaces, using distilled water as a measuring liquid.

As can be seen in Table 1, the pure PVDF showed a contact angle of $83^{\circ}$ while $\theta$ tended to increase with addition of increasing concentrations of HAP in the polymeric matrix. As the micrographs in Figure 1 indicate, this behavior can be attributed to the fact that the mineral load modified the polymer grain formation process. The size and shape of the pores and the surface characteristics were likely modified, reducing the composites' surface wettability. However, in the compositions containing proportions of more than 60/40 the contact angle underwent a strong reduction due to saturation of the polymeric matrix by the mineral load, which caused the appearance of HAP in the form of powder on the surface of the PVDF/HAP films, increasing the material's wettability.

\subsection{Mechanical testing}

Table 2 shows the ultimate strength and total strain of PVDF/HAP film test specimens in the various proportions tested here. The addition of HAP clearly reduced both the strength and the strain of PVDF, increasing the film's brittleness and reducing its mechanical strength, although it retained a certain degree of flexibility. This was observed in the handling of the films when the test specimens were put into the testing machine. The test specimens required extra care when they were fixed to the grips of the testing machine to prevent them from disintegrating during handling. We also found that the flexibility of the films declined as the HAP load in the PVDF polymeric matrix increased. Table 2 shows that, in general, the tensile tests of the irradiated and non-irradiated samples revealed no significant alteration in the samples' mechanical properties. The only alteration occurred in the ultimate toughness of pure PVDF (without added HAP), whose values after irradiation dropped from 45.2 to $33.7 \mathrm{MPa}$. To further illustrate the effect of the addition of HAP on the mechanical properties of PVDF films, Figure 4 shows the stress-strain curves obtained from

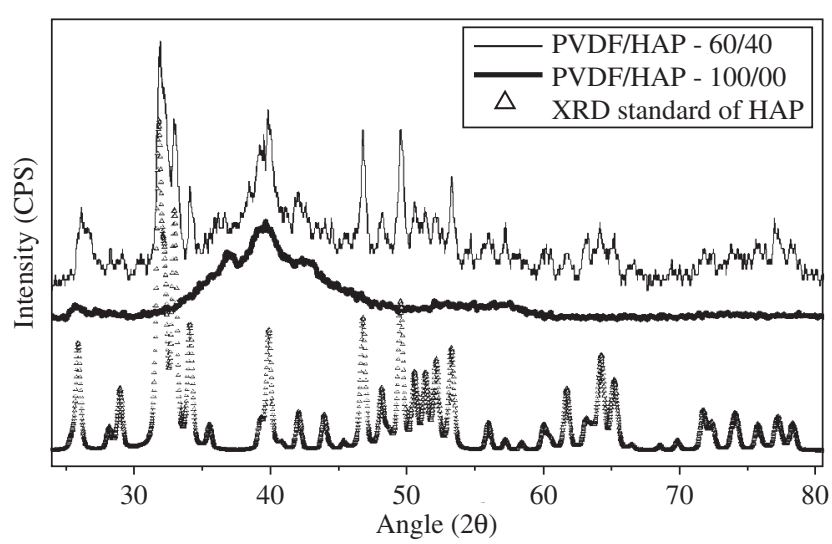

Figure 3. XRD patterns of PVDF/HAP: 100/00 (sliced line), PVDF/HAP - 60/40 (thick line) and HAP phase (upward pointing triangles - PDF n. 86-740). the tensile tests of samples containing PVDF/HAP in proportions of 100/00, 70/30 and 60/40, all non-irradiated.

\subsection{Cytotoxicity assay}

Table 3 shows the percentage of viable cells in PVDF and PVDF/ HAP membrane samples in the serially diluted extracts obtained from the cytotoxicity assay, as well as the negative and positive controls.

The cell viability percentage data compared in the graph against the extract concentration (Figure 5) shows the cell viability curves of the cytotoxicity assay. All the samples tested here presented the same behavior as the negative control, with the viability curves above the cytotoxicity index $\left(\mathrm{IC}_{50 \%}\right)$ line, indicating no toxic effect. On the other hand, the positive control presented a cytotoxic behavior of $\mathrm{IC}_{50 \%}=37$, indicating that the extract from the positive control, in a $37 \%$ dilution, damaged $50 \%$ of the cell population in this assay.

\section{Conclusions}

The maximum limit of HAP incorporated in PVDF polymeric matrix was $40 \%$, forming satisfactorily homogeneous PVDF/HAP composites, as indicated by SEM.

Table 1. Contact angle values $(\theta)$ of the PVDF/HAP composite film surfaces and their respective standard deviations.

\begin{tabular}{ccccccc}
\hline \multicolumn{6}{c}{ PVDF/HAP Composites } \\
\hline $100 / 00$ & $90 / 10$ & $80 / 20$ & $70 / 30$ & $60 / 40$ & $50 / 50$ & $40 / 60$ \\
$83 \pm 2.0^{\circ}$ & $94 \pm 0^{\circ}$ & $98 \pm 0^{\circ}$ & $110 \pm 0^{\circ}$ & $115 \pm 0.5^{\circ}$ & $41 \pm 1.5^{\circ}$ & $19 \pm 3.8^{\circ}$ \\
\hline
\end{tabular}

Table 2. Ultimate strength and total strain obtained from PVDF and PVDF/ HAP tensile test.

\begin{tabular}{lcc}
\hline Proportion PVDF/HAP & $\begin{array}{c}\text { Ultimate strength } \\
(\mathrm{MPa})\end{array}$ & $\begin{array}{c}\text { Total strain } \\
(\%)\end{array}$ \\
\hline 100/00 without irradiation & $45.2 \pm 1.2$ & $11.3 \pm 2.3$ \\
100/00 irradiated & $33.7 \pm 0.3$ & $10.9 \pm 0.3$ \\
70/30 without irradiation & $19.1 \pm 1.1$ & $3.9 \pm 0.4$ \\
70/30 irradiated & $19.0 \pm 2.8$ & $3.6 \pm 0.2$ \\
60/40 without irradiation & $12.8 \pm 1.0$ & $3.5 \pm 0.4$ \\
60/40 irradiated & $10.4 \pm 1.6$ & $3.0 \pm 0.2$ \\
\hline
\end{tabular}

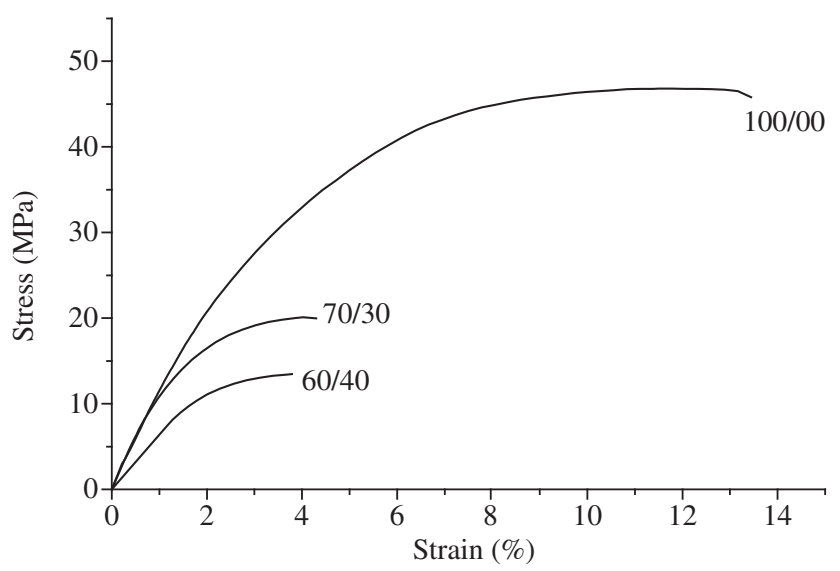

Figure 4. Stress-strain curves obtained from tensile tests of PVDF/HAP in the proportions of 100/00, 70/30 and 60/40. 
Table 3. Percentage of viable cells in PVDF and PVDF/HAP membranes, indicated by the cytotoxicity assay using the neutral red uptake method.

\begin{tabular}{ccccc}
\hline \multirow{2}{*}{$\begin{array}{c}\text { Extract } \\
\text { concentration } \\
(\%)\end{array}$} & $\begin{array}{c}\text { Negative } \\
\text { control }\end{array}$ & $\begin{array}{c}\text { Positive } \\
\text { control }\end{array}$ & $\begin{array}{c}\text { PVDF } \\
\text { membrane }\end{array}$ & $\begin{array}{c}\text { PVDF/HAP } \\
\text { membrane }\end{array}$ \\
\hline 100 & $99 \pm 11$ & $0 \pm 0$ & $105 \pm 6$ & $90 \pm 9$ \\
50 & $107 \pm 6$ & $29 \pm 1$ & $108 \pm 4$ & $101 \pm 8$ \\
25 & $100 \pm 4$ & $68 \pm 12$ & $105 \pm 1$ & $125 \pm 13$ \\
12.5 & $98 \pm 3$ & $89 \pm 14$ & $108 \pm 4$ & $110 \pm 9$ \\
6.25 & $97 \pm 3$ & $89 \pm 6$ & $108 \pm 4$ & $117 \pm 9$ \\
\hline
\end{tabular}

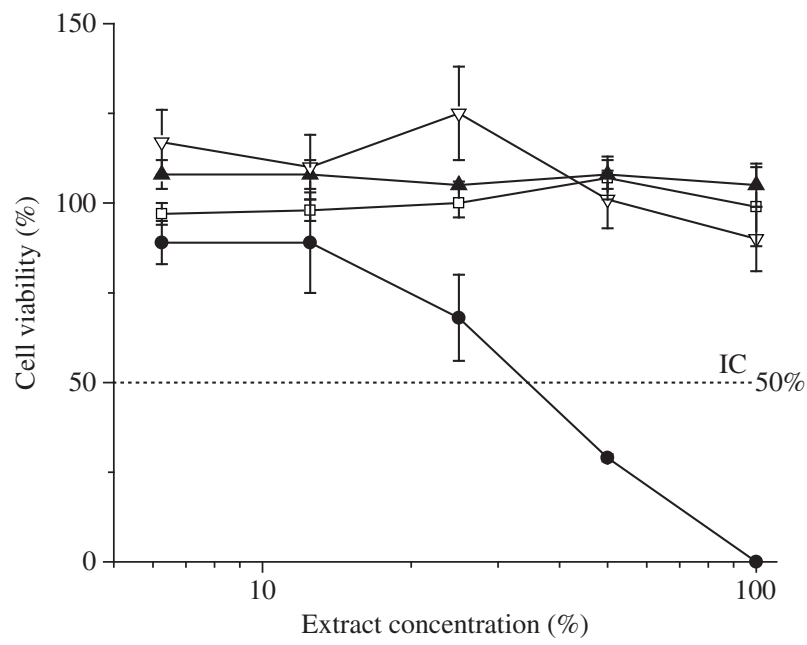

$$
\begin{aligned}
& \longrightarrow \square \text { Negative control } \\
& \longrightarrow \text { Positive control } \\
& \longrightarrow \text { PVDF membrane } \\
& \rightarrow-\text { PVDF/HA membrane }
\end{aligned}
$$

Figure 5. Cell viability curves of PVDF and PVDF/HAP membranes in the cytotoxicity assay using the neutral red uptake method.

The pores size and morphology in the PVDF matrix was modified by the addition of HAP, although the polymer's crystalline structure remained unaltered. This suggests that the incorporated HAP did not react with the PVDF matrix during the synthesization process, i.e., the composite films were not structurally modified. The addition of HAP also rendered the PVDF more brittle and less mechanically resistant, despite a certain degree of flexibility in handling, a characteristic that declined as the load in the polymeric matrix increased.

The surface wettability of PVDF/HAP composites tended to decrease as the percentage in weight of HAP increased in the polymeric matrix. This characteristic may represent an obstacle to the material's application in bone implants, since it is known that the greater the material's wettability the better its interaction with the body's tissues and liquids. However, a definitive conclusion concerning this property can only be reached by means of in vivo tests, which we plan to carry out in future studies.

The PVDF and PVDF/HAP membranes showed no toxicity in the first in vitro assay of the biocompatibility study, and are thus potential candidates for use in medical and dental applications. However, it must be kept in mind that, depending on the application in which these membranes are to be used, other biocompatibility tests must be carried out, in line with the international standard ISO $10993^{15}$.

\section{Acknowledgments}

The authors would like to thank the FISPOL group of the Department of Polymer Technology, Faculty of Chemical Engineering, Campinas State University for its support. We are also indebted to the students Rezolina Pereira dos Santos, from the Adolfo Lutz Institute, and Renata Hage Amaral, from IPEN, for their technical support, and to $\mathrm{CNPq}$ (Brazil) for granting a fellowship.

\section{References}

1. Park JB. Biomaterials Sicience and Engineering. New York: Plenum Press; 1984.

2. Suchanek W, Yoshimura M. Processing and properties of hydroxyapatitebased biomaterials for use as hard tissue replacement implants. Journal of Materials Research. 1998; 13(1):94-117.

3. Inoue k, Ohgushi H, Yoshikawa T, Okumura M, Tamai S, Dohi Y. The effect of aging on bone formation in porous hydroxyapatite: biochemical and histological analysis. Journal of Bone and Mineral Research. 1997; 12(6):989-994.

4. Ikada Y, Shikinami Y, Hara Y, Tagawa M, Fukada E. Enhancement of bone formation by drawn poly(L-lactide). Journal of Biomedical Materials Research. 1996; 30:553-558.

5. Laurencin CT, El-Amin SF, Ibim SE, Willoughby DA, Attawia MA, Allcock HR, Ambrosio AA. A highly porous 3-dimensional polyphosphazene polymer matrix for skeletal tissue regeneration. Journal of Biomedical Materials Research. 1996; 30:133-138.

6. Yaszemki MJ, Payne RG, Hayes WC, Langer R, Mikos AG. Evolution of bone transplantation: molecular, cellular and tissue strategies to engineer human bone. Biomaterials. 1996; 17(2):175-185.

7. Yamazaki Y, Oida S, Ishihara K, Nakabayashi N. Ectopic induction of cartilage and bone by bovine bone morphogenetic protein using a biodegradable polymeric reservoir. Journal of Biomedical Materials Research. 1996; 30:1-4.

8. Buonomenna MG, Drioli E, Licini G, Scrimin P. New catalytic polymeric membranes incorporating Ti(IV) trialkanolamines complexes: Synthesis, characterization, and application in catalysis. Microreactor Technology and Process Intensification acs Symposium. 2005; 914:309-321.

9. Ghosh R. Membrane chromatographic immunoassay method for rapid quantitative analysis of specific serum antibodies. Biotechnology and Bioengineering. 2006; 93(2):280-285.

10. Ghosh R. Fractionation of human plasma proteins by hydrophobic interaction membrane chromatography. Journal of Membrane Science. 2005; 260(1-2):112-118.

11. Pham VC, Henzel WJ, Lill JR. Rapid on-membrane proteolytic cleavage for Edman sequencing and mass spectrometric identification of proteins. Electrophoresis. 2005; 26(22):4243-4251.

12. Chang CY, Liao HK, Juo CG, Chen SH, Chen YJ. Improved analysis of membrane protein by PVDF-aided, matrix-assisted laser desorption/ionization mass spectrometry. Analytica Chimica Acta. 2006; 556(1):237-246.

13. Hu FX, Neoh KG, Cen L, Kang ET. Antibacterial and antifungal efficacy of surface functionalized polymeric beads in repeated applications. Biotechnology and Bioengineering. 2005; 89(4):474-484.

14. Adam NK. Advanced in Chemistry Series. Washington DC: American Chemical Society; 1964.

15. ISO document 10993-5. Biological Evaluation of Medical Devices. Part 5, Tests for Cytotoxicity: in vitro methods; 1992.

16. Rogero SO, Malmonge SM, Lugão AB, Ikeda TI, Miyamaru L, Cruz AS. Biocompatibility study of polymeric biomaterials. Artificial Organs. 2003; 27(5):424-427.

17. Rogero SO, Lugão AB, Ikeda TI, Cruz AS. Teste in vitro de citotoxicidade: estudo comparativo entre duas metodologias. Materials Research. 2003; 6(3):317-320.

18. Sheldon RP. Composite Polymeric Materials. London and New York: Applied Science Publishers; 1982.

19. Lovinger AJ. Development in Cristalline Polymers. London: Edited by D.C. Basset, Published by Applied Science Publishers Ltd.; 1982. p. 195-273. 
